\title{
Wireless Sensor Network : A Survey
}

\author{
Swetha. $\mathbf{R}^{1}$, Santhosh Amarnath. $\mathbf{V}^{2}$, Anitha Sofia.V.S ${ }^{3}$ \\ Department of Computer Technology, Sri Krishna Arts and Science College, Coimbatore, Tamil Nadu, India
}

\begin{abstract}
Wireless Sensor Network the existing cluster based technique may result in increased network works. WSNs are spatially distributed autonomous sensors to monitor physical or environmental conditions, such as temperature, sound, pressure, etc. Sensor networks can contain hundreds or thousands of sensing nodes. The development of wireless sensor networks was motivated by military applications such as battlefield surveillance; Networks are used in many industrial and applications, such as industrial process and control, machine health monitoring.
\end{abstract}

Keywords: Sensor, Terrestrial, Reliability

\section{INTRODUCTION}

Wireless sensor network refers to a group of spatially dispersed and dedicated sensors for monitoring and recording the physical conditions of the environment and organizing the collected data at a central location. Wireless sensor network measure environmental conditions like temperature, sound, pollution levels, wind, etc. Wireless networks in the sense that they reply on wireless connectivity and spontaneous formation of networks so that sensor data can be transported wirelessly. Sensor are smaller, cheaper and intelligent. Wireless sensor networks are distributed sensors to monitor physical or environmental conditions, such as temperature, sound and pressure. The more modern networks are bidirectional, also enabling control of sensor activity. The development of wireless sensor networks was motivated by military applications such as battlefield surveillance; Networks are used in many industrial and applications, industrial process and control, machine health monitoring.

\section{TYPES OF WNS}

Types of WSNs (Wireless Sensor Networks) Depending on the environment, the types of networks are decided so that those can be underwater, underground, land etc. Different types of WSNs include:

$\begin{array}{ll}\text { 1. } & \text { Terrestrial WSNs } \\ \text { 2. } & \text { Underground WSNs } \\ \text { 3. } & \text { Underwater WSNs } \\ \text { 4. } & \text { Multimedia WSNs } \\ \text { 5. } & \text { Mobile WSNs }\end{array}$

1. Terrestrial WSNs: Terrestrial WSNs are capable of communicating base stations efficiently, and consist of hundreds to thousands of wireless sensor nodes deployed either in unstructured or structured manner. In an unstructured mode, the sensor nodes are randomly distributed within the target area that is dropped from a fixed plane. The pre-planned or structured mode considers optimal placement, grid placement, and 2D, 3D placement models.

2. Underground WSNs: The underground wireless sensor networks are more experience, maintenance, and equipment cost is high and planning should be very careful. The WSNs networks consist of a number of sensor nodes that are hidden in the ground to monitor underground conditions. To relay information from the sensor nodes to the base station, additional sink nodes are located above the ground.

3. Under Water WSNs: The $70 \%$ of the earth is occupied with water. These networks consist of a number of sensor nodes and vehicles under water. Uunderwater vehicles are used for gathering data from these sensor nodes. A challenge of underwater communication is a long delay, and bandwidth and sensor failures.

4. Multimedia WSNs: Multimedia wireless sensor networks have been used to enable tracking and monitoring of events in the form of multimedia like image, video, and audio. These networks consist of low-cost sensor nodes with microphones and cameras. These nodes are interconnected with each other over a wireless connection for data compression and data retrieval 
Vol. 7, Issue 11, November 2018

5. Mobile WSNs: Mobile networks consist of a collection of sensor nodes that can be moved on their own and interacted with the physical environment. The mobile nodes have the ability to computer sense and communicate.The mobile wireless sensor networks are much more versatile than the static sensor networks.

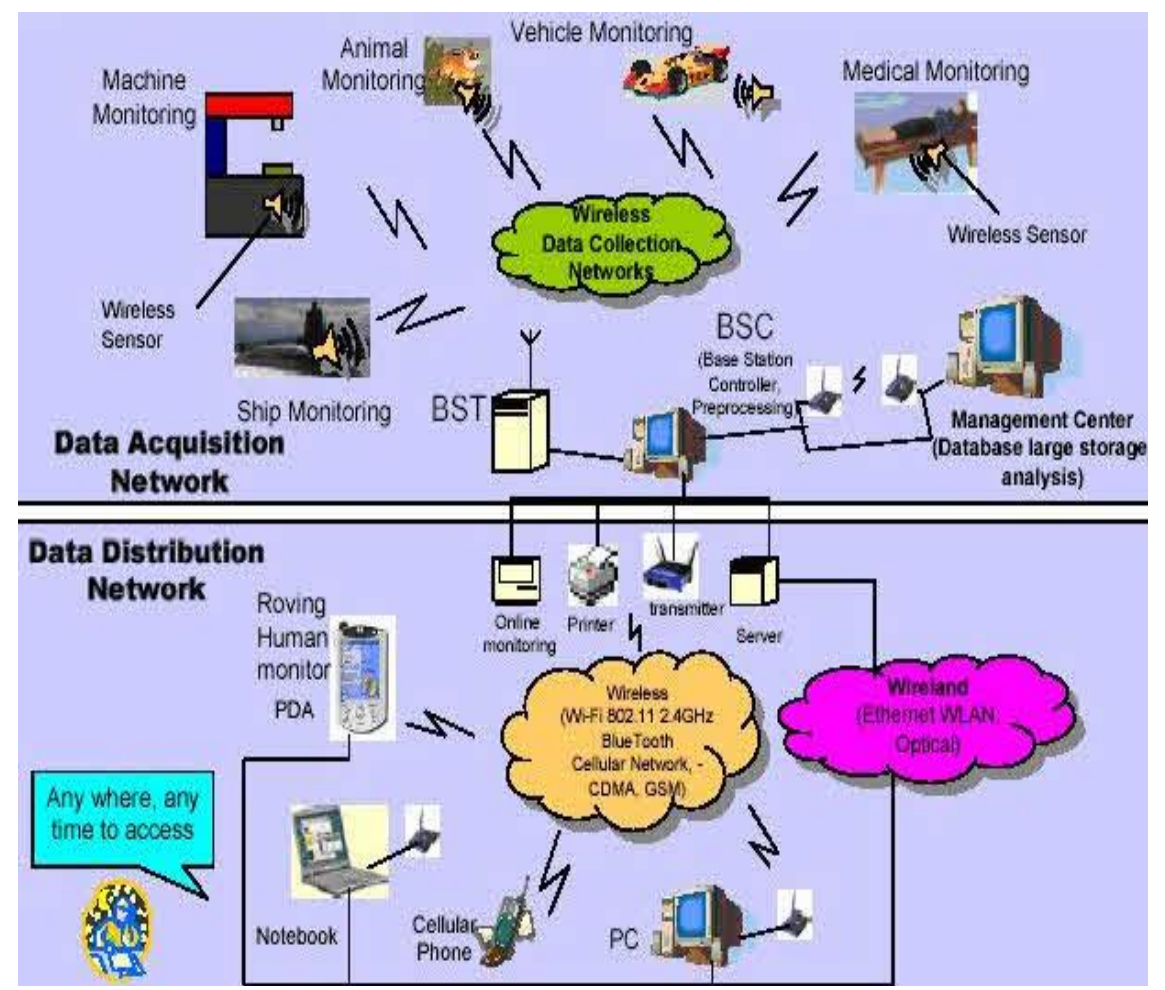

III. WIRELESS SENSOR NETWORKS APPLICATIONS

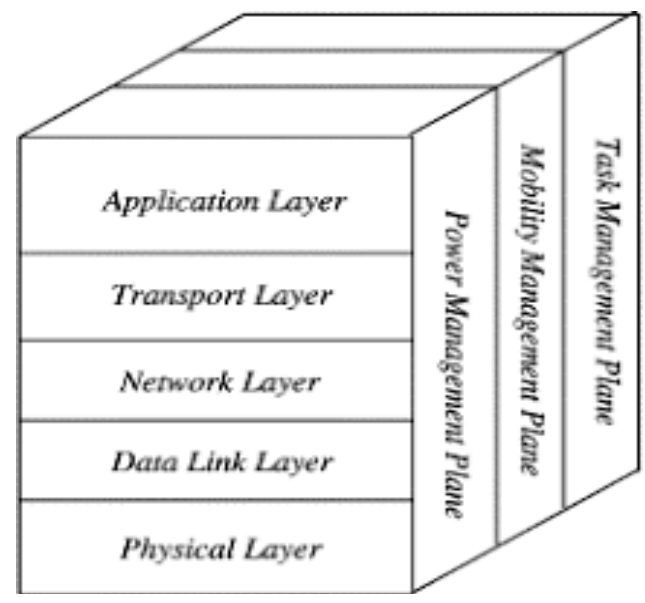

\section{Application Layer}

The application layer is liable for traffic management and offers software for numerous applications that convert the data in a clear form to find positive information. Sensor networks arranged in numerous applications in different fields such as agricultural, military, environment, medical, etc.

\section{Transport Layer}

The function of the transport layer is to deliver congestion avoidance and reliability where a lot of protocols intended to offer this function are either practical on the upstream. These protocols use dissimilar mechanisms for loss recognition and loss recovery. The transport layer is exactly needed when a system is planned to contact other networks. Providing a reliable loss recovery is more energy efficient and that is one of the main reasons why TCP is not fit for WSN. In general, Transport layers can be separated into Packet driven, Event driven. There are some popular protocols in the 
transport layer namely STCP (Sensor Transmission Control Protocol), PORT (Price-Oriented Reliable Transport Protocol and PSFQ (pump slow fetch quick).

\section{Network Layer}

The main function of the network layer is routing, it has a lot of tasks based on the application, but actually, the main tasks are in the power conserving, partial memory, buffers, and sensor don't have a universal ID and have to be selforganized.The simple idea of the routing protocol is to explain a reliable lane and redundant lanes, according to a convinced scale called metric, which varies from protocol to protocol. There are a lot of existing protocols for this network layer, they can be separate into; flat routing and hierarchal routing or can be separated into time driven, querydriven \& event driven.

\section{Data Link Layer}

The data link layer is liable for multiplexing data frame detection, data streams, MAC, \& error control, confirm the reliability of point-point (or) point- multipoint.

\section{Physical Layer}

The physical layer provides an edge for transferring a stream of bits above physical medium. This layer is responsible for the selection of frequency, generation of a carrier frequency, signal detection, Modulation \& data encryption. IEEE 802.15.4 is suggested as typical for low rate particular areas \& wireless sensor network with low cost, power consumption, density, the range of communication to improve the battery life. CSMA/CA is used to support star \& peer to peer topology. There are several versions of IEEE 802.15.4.V.

\section{Advantage:}

- Wireless sensor networks are used in those harsh and hostile environments where wired networks can't be deployed.

- Wireless sensor network is easily operated.

- Wireless sensor network is easily deployed.

- WSN over the static wireless sensor networks include better and improved coverage, better energy efficiency and superior channel capacity

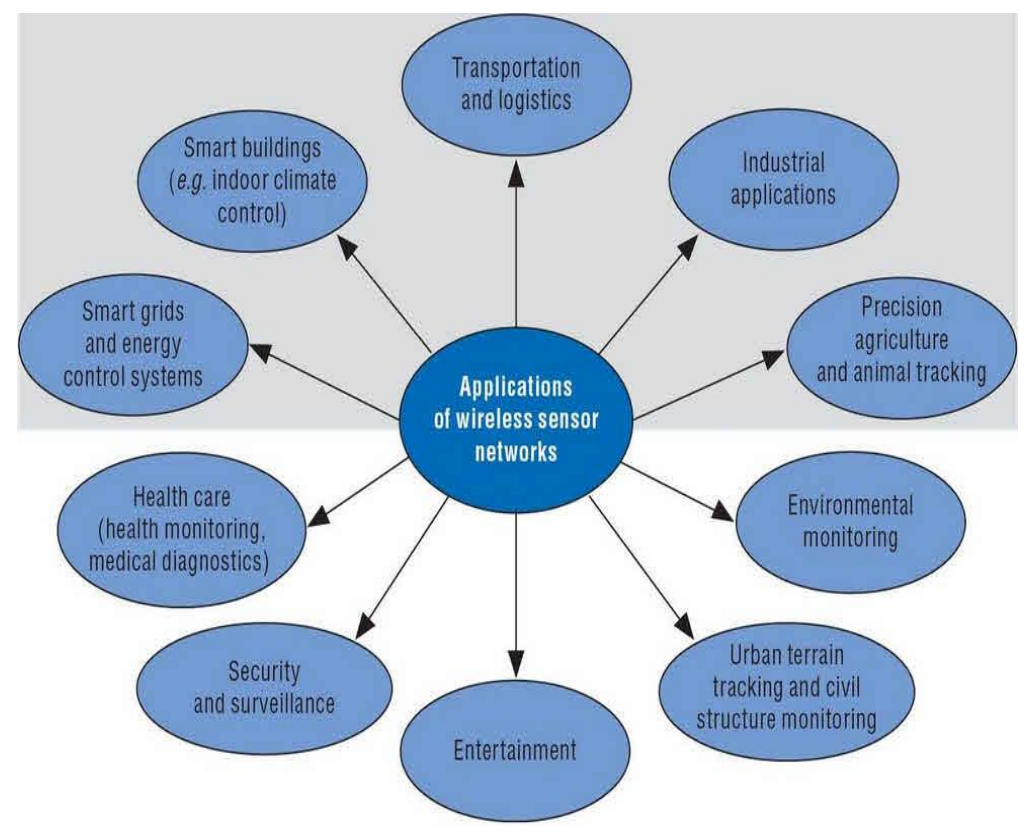

\section{Disadvantages:}

- Limited computation and communication resources are the only disadvantages in wireless sensor networks.

- They have limited battery power, limited storage and computation capabilities, prone to the security attacks and have limited bandwidth to communicate.

\section{Characteristic of wireless sensor networks:}

- $\quad$ The characteristics of WSN include the following.

- The consumption of Power limits for nodes with batteries 


\section{IJARCCE}

- $\quad$ Capacity to handle with node failures

- $\quad$ Some mobility of nodes and Heterogeneity of nodes

- Scalability to large scale of distribution

- Capability to ensure strict environmental conditions

- Simple to use

- Cross-layer design

\section{CONCLUSION}

* WSN needs high level of security due to its harsh environment.

* Wireless sensor network require solution for energy aware and real-time routing, security and nodes.

* Many application can been proposed.

* Wireless sensor networks is mostly used in real time.

\section{REFERENCES}

[1]. https://www.elprocus.com/introduction-to-wireless-sensor-networks-types-and-applications/

[2]. https://en.m.wikipedia.org/wiki/Wireless_sensor_network

[3]. https://scholar.google.co.in/scholar?hl=en\&as_sdt=0\%2C5\&q=wireless+sensor+network+survey\&oq=wireless+sensor+\#d=gs_qabs\&p=\&u=\% 23p\%3DhVitWqzytK8J

[4]. https://www.sciencedirect.com/science/article/abs/pii/S1389128608001254

[5]. https://www.elprocus.com/introduction-to-wireless-sensor-networks-types-and-applications/

[6]. http://www.ni.com/white-paper/7142/en/

[7]. Adaptive Protocols for Information Dissemination in Wireless Sensor Networks by Wendi Rabiner Heinzelman, Joanna Kulik, and Hari Balakrishnan

[8]. Wireless Sensor Networks by Lakshmi Ramaswamy.

[9]. Support in Wireless Sensor Networks: A Survey, by Dazhi Chen and Pramod K. Varshney.

[10]. A Survey on Sensor Networks, http://www-net.cs.umass.edu/cs791_sensornets/papers/akyildiz2.pdf (105,362 Bytes) 